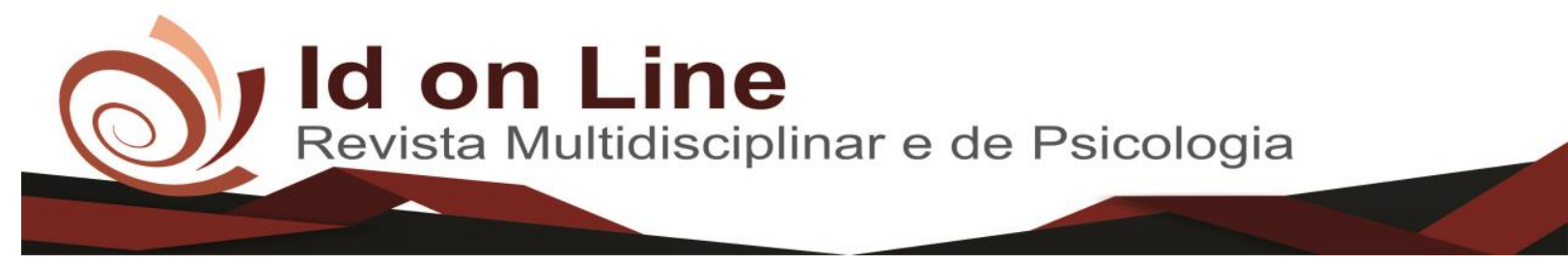

Artigo

\title{
A Hidroterapia no Tratamento de Indivíduos com Fibromialgia
}

\author{
Jaqueline Santos da Silva ; Auricéia Ferreira Souto Spósito² ; Carla Pequeno da Silva ${ }^{3}$
}

\begin{abstract}
Resumo: Fibromialgia é uma patologia de etiologia desconhecida que acomete principalmente as mulheres, seguido de crianças e idosos e se caracteriza por dores musculares difusas que podem estar associadas a distúrbios do sono, fadiga crônica e distúrbios psíquicos e intestinais ou em grupos. O objetivo desta pesquisa foi verificar a eficácia da hidroterapia no alívio de dores e na qualidade do sono de indivíduos com fibromialgia. Trata-se de um estudo do tipo ensaio clínico, descritivo e exploratório, com delineamento transversal e abordagem quantitativa, desenvolvido no município de Vitória da Conquista, Bahia. A população do estudo foi representada por 13 indivíduos do sexo feminino com fibromialgia, cadastrados no Núcleo de Estudos em Fisioterapia de uma instituição privada. Inicialmente foi aplicado um questionário sociodemográfico acrescido da avaliação da qualidade do sono através do questionário de Índice de Qualidade de Sono de Pittsburgh (IQSP) e para mensurar a intensidade de dores a Escala Visual Analógica (EVA). Após isso, deu-se início a intervenção composta por exercícios no meio aquático. Por fim o questionário de Pittsburgh (IQSP) e a Escala Visual Analógica (EVA) foram novamente aplicados nos indivíduos. Conclui-se, após a comparação dos dados, que a hidroterapia é eficaz enquanto tratamento para a fibromialgia, melhorando a qualidade de sono e agindo para o alívio de dores.
\end{abstract}

Palavras-Chave: Fibromialgia, hidroterapia, dor, qualidade de sono.

\section{The Hydrotherapy in the Treatment of Individuals with Fibromyalgia}

\begin{abstract}
Fibromyalgia is a pathology of unknown etiology that mainly affects women, followed by children and the elderly and is characterized by diffuse muscular pains that may be associated with sleep disorders, chronic fatigue, and psychic and intestinal disorders or in groups. The objective of this research was to verify the efficacy of hydrotherapy in relieving pain and sleep quality of individuals with fibromyalgia. This is a descriptive and exploratory clinical study, with a cross-sectional design and a quantitative approach, developed in the city of Vitória da Conquista, Bahia. The study population was represented by 13 female individuals with fibromyalgia, enrolled in the Nucleus of Studies in Physiotherapy of a private institution. Initially, a sociodemographic questionnaire plus sleep quality assessment was applied through the Pittsburgh Sleep Quality Index (IQSP) questionnaire and to measure the intensity of pain in the Visual Analogue Scale (VAS). After this, the intervention composed of exercises in the aquatic environment began. Finally, the Pittsburgh questionnaire (IQSP) and Visual Analogue Scale (EVA) were applied again in the individuals. It is concluded, after data comparison, that hydrotherapy is effective as a treatment for fibromyalgia, improving the quality of sleep and acting for the relief of pain.
\end{abstract}

Key words: Fibromyalgia, hydrotherapy, pain, quality of sleep.

\footnotetext{
${ }^{1}$ Discente do curso de Fisioterapia da Faculdade Independente do Nordeste - FAINOR, Vitória da Conquista/ BA. E-mail: Jack_litza23@ @otmail.com.br

${ }^{2}$ Fisioterapeuta, Especialista em Traumato-Ortopedia, Docente na Faculdade Independente do Nordeste - FAINOR, Vitória da Conquista/ BA. E-mail: aurissouto@gmail.com

${ }^{3}$ Fisioterapeuta, Mestra em Saúde Pública, Docente na Faculdade Independente do Nordeste - FAINOR, Vitória da Conquista/ BA. E-mail:carla_rivka@hotmail.com
}

198 Id on Line Rev. Mult. Psic. V.12, N. 42, Supl. 1, p. 198-210, 2018 - ISSN 1981-1179 198 Edição eletrônica em http://idonline.emnuvens.com.br/id 


\section{Introdução}

A fibromialgia (FM) é uma síndrome crônica do foro reumático, não articular, cujos fatores etiológicos não estão bem compreendidos. Se esta condição estiver associada a outras doenças, denomina-se FM secundária, se não designa-se FM primária. A FM atinge cerca de 2\% da população adulta. São fatores de risco: a) o sexo (as mulheres são 5 a 9 vezes mais afetadas do que os homens); b) a idade (inicia-se entre os 20 e os 50 anos). As crianças e jovens também podem sofrer de FM, mas durante a idade escolar a frequência é igual em ambos os sexos. Os sintomas caracterizam-se pela presença de dor musculoesquelética generalizada e dor excessiva nos tender points - locais específicos do corpo que causam dor leve a moderada em indivíduos saudáveis. A dor presente na FM é considerada crónica, uma vez que apresenta uma duração superior a três meses (SILVA, 2014).

Entre outros sintomas destacam-se a fadiga, fraqueza muscular, rigidez matinal, sensação de edema, perturbações do sono, ansiedade, depressão, alterações de humor, parestesias, dificuldade de concentração, défice de memória, cefaleias e problemas gastrointestinais, afetando, deste modo, a qualidade de vida dos utentes. A sintomatologia desta síndrome pode variar de acordo com as condições meteorológicas, ou seja, com o frio e a humidade podem agravar, e com o calor aliviar. Os indivíduos com FM apresentam uma redução da eficácia cardiovascular, da capacidade aeróbia, da circulação periférica e, consequentemente, uma redução do desempenho físico geral. Esta situação pode ser agravada por alterações posturais que provoquem o encurtamento dos músculos respiratórios, reduzindo a expansão pulmonar (BATISTA, BORGES e WIBELINGER, 2012).

Dentre os possíveis tratamentos indicados para a fibromialgia encontra-se a Hidroterapia a qual é um recurso fisioterapêutico que tem demonstrado bons resultados no tratamento e prevenção de diversas patologias ortopédicas, neurológicas e pediátricas, entre outras. Uma variedade de métodos aquáticos vem surgindo constantemente, cujos efeitos combinam os produzidos pelas propriedades da água aos produzidos pelos exercícios terapêuticos (HIDALGO et al., 2013).

O calor e a pressão hidrostática da água permitem reduzir o estímulo nociceptivo, por meio da estimulação dos mecanorreceptores e dos receptores térmicos. Para além disso, o 
calor também aumenta o fluxo sanguíneo, o que reverte a isquemia nos tecidos e promove a remoção dos mediadores químicos da inflamação, facilitando o relaxamento muscular. Por sua vez, a pressão hidrostática, ao facilitar o retorno venoso pode aliviar a dor pela redução do edema periférico e pela inibição do sistema nervoso simpático. Também a facilidade do movimento na água pode ativar as vias supra espinhais, o que resulta na redução da intensidade da dor (BUYSSE, REYNOLDS, MONK, BERMAN, e KUPFER, 1989).

Esta modalidade terapêutica não apresenta nenhuma contraindicação para a FM, exceto as comuns a todas as condições, como insuficiência cardíaca e respiratória, alterações na tensão arterial, incontinência e infecções urinárias, incontinência fecal, infecções cutâneas e alergias aos componentes químicos da água (BRAZ, et al, 2011).

Para o tratamento dos indivíduos com FM, as intervenções escolhidas vão depender da extensão e da gravidade dos sintomas, bem como da motivação, participação e dos fatores psicossociais (BATISTA, BORGES e WIBELINGER, 2012).

Sabendo que a intervenção fisioterapêutica gera benefícios a qualquer indivíduo independente da sua faixa etária, tornou-se interessante identificar o quanto é a eficácia de um programa de intervenção voltada a melhora das pessoas com fibromialgia.

O presente estudo teve por objetivo verificar eficácia da hidroterapia no alívio de dores e na qualidade do sono de indivíduos com fibromialgia.

\section{Materiais e Métodos}

Trata-se de um estudo tipo ensaio clínico, descritivo e exploratório, com delineamento transversal e abordagem quantitativa.

A pesquisa foi realizada em um Núcleo de Estudos em Fisioterapia de uma instituição privada, localizada no município de Vitória da Conquista - BA. Vinte e um sujeitos estavam cadastrados, sendo todos do sexo feminino, entretanto, apenas treze sujeitos participaram da pesquisa. Os outros sete indivíduos foram eliminados devido à problemas dermatológicos e/ou não disponibilidade de tempo, critérios de exclusão deste trabalho. Os sujeitos responderam duas vezes ao questionário e a escala: antes de participar do protocolo de tratamento e logo após imediatamente ao término das sessões. A coleta de dados foi feita pelo mesmo avaliador nas duas etapas. 
A idade média dos indivíduos, foi de 53 anos, predominantemente negras ou pardas $(92,31 \%)$, com renda familiar superior a um e inferior a dois salários mínimos. No que diz respeito a estrutura familiar, $69,23 \%$ eram casadas enquanto $30,77 \%$ viúvas ou solteiras. Dividiam a residência com outros 3 moradores, em média.

Para mensurar a intensidade da dor, foi utilizada a Escala Visual Analógica (EVA), essa consiste em auxiliar na aferição da intensidade da dor no paciente, é um instrumento importante para verificarmos a evolução do paciente durante o tratamento e mesmo a cada atendimento, de maneira mais fidedigna. Também é útil para podermos analisar se o tratamento está sendo efetivo, quais procedimentos têm surtido melhores resultados, assim como se há alguma deficiência no tratamento, de acordo com o grau de melhora ou piora da dor.

A EVA pode ser utilizada no início e no final de cada atendimento, registrando o resultado sempre da evolução. Para utilizar a EVA o atendente deve questionar o paciente quanto ao seu grau de dor sendo que 0 significa ausência total de dor e 10 o nível de dor máxima suportável pelo paciente.

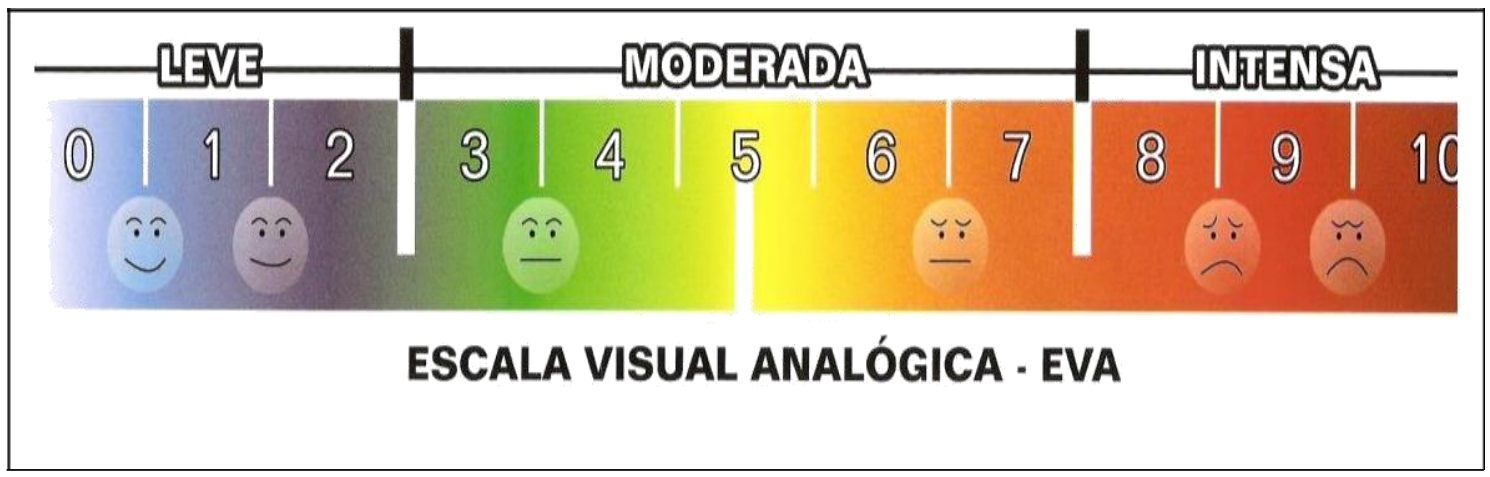

Figura 01 Escala Visual Analògica - EVA

Fonte: BRASIL, Ministério da Saúde. Instituto Nacional de Câncer. Cuidados Paliativos Oncológicos: controle da dor. Rio de Janeiro: INCA, 2001. Disponível em: http://bvsms.saude.gov.br/bvs/publicacoes/inca/manual_dor.pdf

Para avaliar a qualidade do sono, foi utilizado o questionário de Índice de Qualidade de Sono de Pittsburgh (IQSP). Este questionário avalia a qualidade e perturbações do sono durante o período de um mês. Sendo padronizado, simples e bem aceito pelos pacientes. $\mathrm{O}$ mesmo foi traduzido e adaptado para o Brasil por Bertolazzi (2008). 
O protocolo de tratamento foi aplicado duas vezes por semana, durante os meses de setembro e outubro de 2018, sendo composto por 10 sessões de 45 minutos cada, em meio aquático e de forma coletiva, respeitando os limites e a evolução de cada indivíduo. Antes de cada sessão de hidrocinesioterapia foi aferida a pressão arterial de cada paciente.

Cada sessão do programa terapêutico foi composta por quatro fases: Primeira fase Aquecimento de forma global com caminhada por toda extensão da piscina nas seguintes direções: para frente, para trás e com passada lateral com tempo (5 minutos); Segunda fase Alongamento muscular dos membros superiores, inferiores mantido por 20 segundos, com tempo (15 minutos); Terceira fase - exercícios ativos livres para membros superiores e inferiores, inicialmente será realizado sem carga externa e evoluindo para exercícios com utilização de espaguetes e pesos aquáticos, os quais podem variar de $0,5-1 \mathrm{~kg}$, realizando 4 séries com 10 repetições, com tempo (15 minutos); Quarta fase - Relaxamento, com alongamentos ativos de membros inferiores, superiores e cadeia posterior e anterior ao tronco, sendo associando-os a exercícios respiratórios que iram promover relaxamento de forma evolutiva, com tempo (10 minutos).

O estudo iniciou após a aprovação pelo Comitê de Ética da Instituição sob o número do parecer: 2.793.410,e após todas as pacientes assinarem o termo de consentimento livre e esclarecido (TCLE). A análise dos dados se deu da seguinte forma: apresentação da frequência absoluta e frequência relativa dos dados e comparação percentual dos dados anteriores e posteriores a intervenção.

\section{Resultados}

Apresentar-se-ão, neste tópico, os resultados comparativos entre as respostas do Índice da qualidade do sono de Pittsburgh (IQSP) e das respostas da Escala Visual Analógica (EVA).

A reação à EVA no início da pesquisa se deu da seguinte forma:

a) Nove sujeitos $(69,23 \%)$ responderam DOR INTENSA classificada em 10.

b) Quatro sujeitos $(30,67 \%)$ responderam DOR INTENSA classificada em 8 .

Pode-se assumir a média aritmética da amostra de resposta à escala em 9,4 no espectro da DOR INTENSA. 
Sobre o questionário IQSP obtivemos os seguintes resultados anteriores ao tratamento:

A primeira questão é sobre a hora usual que o paciente vai se deitar. $\mathrm{O}$ grupo respondeu da seguinte maneira: $21: 00-6$ pacientes $(46,15 \%) ; 22: 00-4$ pacientes $(30,76 \%) ; 23: 00-3$ pacientes $(23,07 \%)$. Pode-se estabelecer a média da amostragem em 22:00.

A questão de número dois pergunta sobre a quantidade de tempo que o sujeito demora para pegar no sono, para dormir: a latência do sono. Nesse aspecto, as respostas foram: 60min -3 pacientes $(23,07 \%)$; 90 min. -4 pacientes $(30,76 \%), 120 \mathrm{~min}-6$ pacientes $(46,15 \%)$. A média é de 97 minutos.

O próximo questionamento é a respeito do horário usual em que o paciente se levanta. Na questão três, obtiveram-se os seguintes dados: 05:00 - 4 pacientes (30,76\%); 6:00 - 6 pacientes $(46,15 \%) ; 7: 00-3$ pacientes $(23,07 \%)$. Com média em 6:00h.

A questão quatro reúne os dados sobre a quantidade de horas de sono por noite que o sujeito tem, em média. Colheram-se os seguintes dados: 5:30h - 6 pacientes $(46,15 \%) ; 5: 00 \mathrm{~h}-$ 4 pacientes $(30,76 \%) ; 6: 00 \mathrm{~h}-3$ pacientes $(23,07 \%)$. A média é de $5: 30 \mathrm{~h}$.

Quadro 1: Dados pré-intervenção. Vitória da Conquista-BA,2018

\begin{tabular}{|c|l|l|l|l|}
\hline Questão & \multicolumn{1}{|c|}{ Resposta (R1) } & \multicolumn{1}{|c|}{ Resposta (R2) } & \multicolumn{1}{|c|}{ Resposta (R3) } & \multicolumn{1}{|c|}{ Média } \\
\hline 1 & $21: 00(46,15 \%)$ & $22: 00(30,76 \%)$ & $23: 00(23,07 \%)$ & $22: 00 \mathrm{~h}$ \\
\hline 2 & $60 \min (23,07 \%)$ & $90 \min (30,76 \%)$ & $120 \min (46,15 \%)$ & $97 \mathrm{~min}$ \\
\hline 3 & $05: 00(30,76 \%)$ & $06: 00(46,15 \%)$ & $07: 00(23,07)$ & $06: 00 \mathrm{~h}$ \\
\hline 4 & $05: 30(46,15 \%)$ & $05: 00(30,76 \%)$ & $06: 00(23,07 \%)$ & $05: 30 \mathrm{~h}$ \\
\hline
\end{tabular}

Fonte: Elaboração própria.

O IQSP segue da questão cinco até a questão de número dez apontando respostas que podem varias entre 0 e 3, podendo somar um total de 60 pontos. Quanto maior a quantidade de pontos, menor a qualidade do sono. O total absoluto possível dessa pesquisa foi de 780 pontos. Na primeira etapa, todos os sujeitos pontuaram 707 pontos. Uma média de 54,39 pontos/sujeito, ou $90,65 \%$ dos pontos possíveis.

Vale ressaltar que $100 \%$ dos indivíduos responderam que sentiram dores durante a noite três vezes ou mais por semana no último mês e qualificaram a qualidade do próprio sono como 
muito ruim. Todos os indivíduos que participaram da pesquisa também alegaram acordar no meio da noite, de madrugada ou muito cedo três vezes ou mais por semana.

Quadro 2: Cenário geral pré-intervenção. Vitória da Conquista-BA,2018

\begin{tabular}{|c|c|}
\hline Item & Resultado \\
\hline Horário usual de dormir (em média) & $22: 00 \mathrm{~h}$ \\
\hline Latência do sono (em média) & 97 minutos \\
\hline Horário usual de acordar (em média) & 06:00h \\
\hline Horas de sono por noite (em média) & $05: 30 \mathrm{~h}$ \\
\hline Pontos no IQSP (n) & 707 \\
\hline Pontos no IQSP (média por sujeito / \%) & $54,39 / 90,65 \%$ \\
\hline Qualidade do sono & Muito Ruim (100\%) \\
\hline \multirow{2}{*}{$\begin{array}{l}\text { Drntan n EV } / \wedge \\
\text { Fonte: Elaboração própria. } \\
\text { Escala de UUK }\end{array}$} & 9,4 \\
\hline & Dor Intensa \\
\hline
\end{tabular}

Sendo assim, podemos dizer que antes da intervenção, os indivíduos apresentavam o seguinte cenário:

Após a coleta dos primeiros dados, os sujeitos foram submetidos as seções de hidrocinesioterapia. Após as seções, apresentaram-se os seguintes dados:

A reação à EVA, após a intervenção, foi a seguinte:

c) Sete sujeitos $(53,85 \%)$ responderam DOR MODERADA classificada em 6.

d) Seis sujeitos $(46,15 \%)$ responderam DOR MODERADA classificada em 5.

Pode-se assumir a média aritmética da amostra de resposta à escala em 5,5 no espectro da DOR MODERADA.

Quanto ao que diz respeito a hora usual de dormir, os pacientes responderam da seguinte maneira: $21: 00-6$ pacientes $(46,15 \%) ; 22: 00-7$ pacientes $(53,85 \%)$. Pode-se estabelecer a média da amostragem em 21:30.

Sobre a latência do sono, os sujeitos responderam: 40min - 3 pacientes $(23,07 \%)$; 60 min. -4 pacientes $(30,76 \%), 90 \mathrm{~min}-6$ pacientes $(46,15 \%)$. A média é de 69 minutos. 
Quadro 3: Cenário pós intervenção. Vitória da Conquista-BA,2018

\begin{tabular}{|l|l|}
\hline \multicolumn{1}{|c|}{ Item } & \multicolumn{1}{c|}{ Resultado } \\
\hline Horário usual de dormir (em média) & $21: 30 \mathrm{~h}$ \\
\hline Latência do sono (em média) & 69 minutos \\
\hline Horário usual de acordar (em média) & $06: 30 \mathrm{~h}$ \\
\hline Horas de sono por noite (em média) & $06: 15 \mathrm{~h}$ \\
\hline Pontos no IQSP (n) & 482 \\
\hline Pontos no IQSP (média por sujeito / \%) & $37,08 / 61,80 \%$ \\
\hline Qualidade do sono & Boa $(84,61 \%) /$ Ruim $(15,38 \%)$ \\
\hline Pontos na EVA & 5,5 \\
\hline Escala de DOR & Dor Moderada \\
\hline
\end{tabular}

Fonte: Elaboração própria.

Sobre o horário usual de acordar, as respostas foram: 6:00 - 10 pacientes (76,92\%); 7:00 - 3 pacientes $(23,07 \%)$. Com média em 6:30h.

Já no que diz respeito ao tempo de sono, a amostra apresentou os seguintes dados: 6:00 - 10 pacientes $(76,92 \%) ; 6: 30$ - 3 pacientes $(23,07 \%)$. A média é de $6: 15 \mathrm{~h}$.

Houve certa mudança na frequência de dores por semana: 100\% dos indivíduos responderam que sentiram dores durante a noite duas vezes por semana no último mês. 84,61\% qualificaram a qualidade do próprio sono como boa e 15,38\% como ruim. Todos os sujeitos somaram 482 pontos no IQSP, uma média de 37,08 pontos por indivíduo.

Nesse caso, pode-se obter o seguinte cenário pós intervenção:

Ao comparar os cenários pré e pós intervenção pode-se atingir os seguintes dados relativos ao IQSP: Uma redução de 30 minutos na hora de dormir, uma redução de aproximadamente 28 minutos na latência do sono, um aumento de 30 minutos no horário usual de acordar, um ganho de 45 minutos no total do tempo de sono por noite, além da redução de 225 pontos no total de pontos do questionário, o que reflete em um aumento de $28,85 \%$ na taxa de qualidade de sono.

Sobre a qualificação do próprio sono, $84,61 \%$ dos indivíduos saltaram de Muito Ruim para Bom, enquanto 15,38\% mudaram de Muito Ruim para Ruim. 
A tabela 4 apresenta o comparativo desses dados:

Tabela 4: Comparação entre cenários pré e pós intervenção. Vitória da Conquista -BA,2018.

\begin{tabular}{l|l|l|l}
\hline Item & Pré & Pós & Diferença \\
\hline Horário usual de dormir (em média) & $22: 00 \mathrm{~h}$ & $21: 30 \mathrm{~h}$ & $-30 \mathrm{~min}$ \\
\hline Latência do sono (em média) & $97 \mathrm{~min}$ & $69 \mathrm{~min}$ & $-28 \mathrm{~min}$ \\
\hline Horário usual de acordar (em média) & $06: 00 \mathrm{~h}$ & $06: 30 \mathrm{~h}$ & $+30 \mathrm{~min}$ \\
\hline Horas de sono por noite (em média) & $05: 30 \mathrm{~h}$ & $06: 15 \mathrm{~h}$ & $+45 \mathrm{~min}$ \\
\hline Pontos no IQSP (n) & 707 & 482 & 225 \\
\hline Pontos no IQSP (média por sujeito / \%) & $54,39 / 90,65 \%$ & $37,08 / 61,80 \%$ & $17,31 / 28,85 \%$ \\
\hline
\end{tabular}

Fonte: Elaboração própria. 2018.

De acordo com a escala EVA, nosso outro instrumento de análise, houve redução significativa na dor após a intervenção, pois, no cenário pré-intervenção, a classificação geral de dor estava em 9,4, no espectro de dor intensa. Logo após o tratamento, os sujeitos classificaram a dor como 5,5, dentro do espectro de dor moderada. Obtemos o seguinte resultado gráfico:

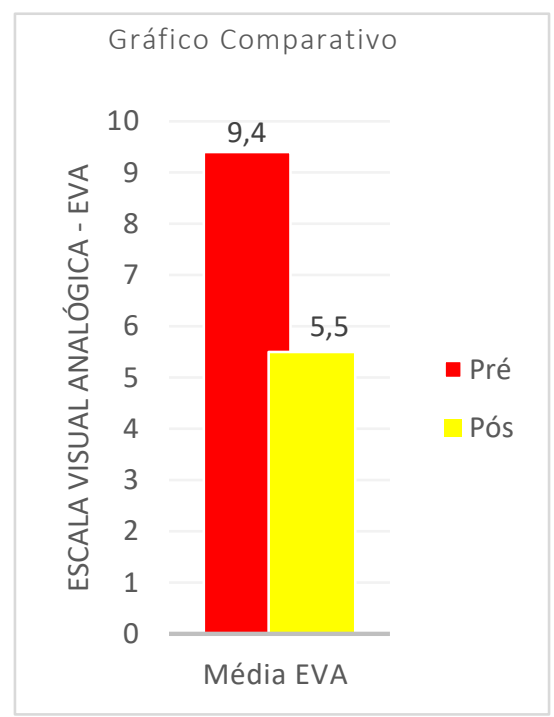

Gráfico 1: Comparativo da Eva pré e pós intervenção. Vitória da Conquista $\mathrm{Ba}, 2018$.

Fonte: Elaboração própria.

\section{Discussão}


Pessoas diagnosticadas com fibromialgia caracterizam-se por apresentar sensibilidade exagerada no corpo, normalmente relatam dificuldade em relacionar a característica e localização da dor, na maioria das vezes são inúmeras áreas dolorosas. Frequentemente apresentam também alguns sintomas associados, como fadiga, dor de cabeça, adormecimento das mãos e pés, alterações do sono, comprometimento das capacidades e habilidades físicas, especialmente redução nas tarefas diárias e força muscular reduzida. Além disso, características psicológicas como ansiedade, depressão e estresse também são apresentadas nestes casos (HIDALGO, 2013)

A hidroterapia trabalha com exercícios aeróbicos que liberam neurotransmissores entre eles a endorfina, proporcionando melhora da memória; melhora do humor; aumento da força muscular; melhora do equilíbrio; aumento da resistência ao exercício; aumento da disposição física e mental; melhora do sistema imunológico e melhora da circulação sanguínea (SILVA, 2014).

$\mathrm{Na}$ fibromialgia, a perturbação de sono dos indivíduos é associada com maior dor e gravidade dos sintomas em geral (por exemplo, fadiga), como também maior rigidez matinal. A fibromialgia foi descrita associada, a distúrbios do sono como síndrome de apnéia do sono tipo obstrutivo, mioclonias noturnas (movimentos periódicos dos membros) e narcolepsia (FERRO, 2005).

Acredita-se que o processo da dor, e esta amplificação dolorosa, possam desequilibrar os mediadores do Sistema Nervoso Central (SNC) e isso faz com que reduza os trabalhos da seretonina, provocando um aumento na produção da substância $\mathrm{P}$ e a diminuição do triptofano e da nerepinefrina. A substância p é um neurotransmissor neuropeptídeo composto por uma cadeia de 11 resíduos de aminoácidos que atua como neuromodulador. A substância $\mathrm{P}$ facilita processos inflamatórios, vômito, ansiedade e nocicepção (resposta a dor). Pode ser encontrado tanto no sistema nervoso central quanto no periférico (SILVA, 2014).

O tratamento é sintomático com medidas farmacológicas e não farmacológicas. O objetivo do tratamento é melhorar a qualidade de vida, restabelecer o equilíbrio emocional, promover alivio da dor, melhorar o condicionamento físico e da fadiga, buscar tratamento específico para as desordens associadas e também é importante informar o paciente e a família sobre os problemas reais que a patologia traz (BARROS, 2015). 
O tratamento farmacológico para a fibromialgia tem como intuito, induzir o sono de melhor qualidade, podendo ser obtido com a utilização de medicamentos como a ciclobenzaprina ou amitriptilina em doses baixas, e também analgésicos e relaxantes musculares. O medicamento não deve ser considerado a única forma ou estratégia para o controle da dor dos pacientes, mas deve ser tratada de forma global, combinando a qualidade de vida, fisioterapia e terapias. Alguns medicamentos atuam no SNC sendo útil para a insônia, dor e fadiga. Existem vários medicamentos sendo utilizado para o tratamento da FM como: Antidepressivos tricíclicos, o ciclobenzaprina como relaxante muscular, o anestésico Lidocaína pode melhorar os níveis da dor. O ácido acetilsalicílico e outros AINES podem auxiliar no controle da dor (ÁLVARES e LIMA, 2010).

Cerca de $90 \%$ dos indivíduos com fibromialgia sentem uma fadiga de moderada a severa, com perda da energia, diminuição da resistência aos exercícios, ou um cansaço semelhante àquele resultante de uma gripe ou perda de sono. Algumas vezes a fadiga é um problema maior do que a dor. Muitas pessoas com fibromialgia experimentam um distúrbio do sono, em que elas não acordam aliviadas, mas sentindo-se cansadas. Pesquisas relatam que o hormônio do crescimento, 80 \% do qual é produzido durante a fase delta do sono, tem um efeito direto na qualidade de manutenção e regeneração dos músculos, e que quando deficiente devido a perturbações do sono, pode, pelo menos em parte, levar aos sintomas musculares da fibromialgia (FERRO, 2005).

Os dados deste estudo, no entanto, apresentam a hidroterapia enquanto um importante mecanismo que pode ser eficaz no tratamento de sujeitos com FM. Ao passo que a qualidade do sono do sujeito melhora, os efeitos dos sintomas se tornam menos agressivos. Se constante, a hidroterapia pode melhorar a qualidade de vida dos sujeitos e ser um potente aliado para o tratamento da FM.

\section{Conclusões}

Pode-se concluir após a análise comparativa dos dados que a hidroterapia é um recurso funcional para a melhoria da qualidade do sono dos sujeitos com Fibromialgia, além de ser um potente recurso de melhoria da dor, e nesse sentido que o mesmo contribui enquanto apresenta a hidroterapia como possibilidade terapêutica para os sujeitos com FM. 
Sendo assim, o presente estudo surgiu com a finalidade de enriquecer os conhecimentos científicos sobre a Hidroterapia como tratamento para a fibromialgia, sendo de fundamental importância para os profissionais que atendem esses pacientes.

\section{Referências}

ÁLVARES, T. T.; LIMA, M. E. A. Fibromialgia: interfaces com as LER/DORT e considerações sobre sua etiologia ocupacional. Ciênc. Saúde coletiva, Rio de Janeiro, v.15, n.3, 2010 .

AZEVEDO, P. W.; BRITO, L. C. N. Efeitos da Hidrocinesioterapia Associada Crioterapia na Gonartrose - Um estudo de caso. Ensaios e Ciência Agrárias e da Saúde, Campo Grande, v.16, n.1, 2012.

BARROS, T. R. M.; et al. Perfil de participantes do projeto "Hidroterapia, exercícios aeróbios e alongamento no tratamento de portadores de fibromialgia". $8^{\circ}$ Congresso de Extensão Universitária da UNESP, 2015.

BATISTA, J. S.; BORGES, A. M.; WIBELINGER, L. M. Tratamento Fisioterapêutico na Síndrome da dor Miofascial e Fibromialgia. Rev Dor. São Paulo, v. 13, n.2, 2012.

BATISTA, T.; ALFAIATE, V.; SILVA, V.; GOMES, M.; ALVES, S. Os efeitos da hidroterapia na fibromialgia - revisão de literatura. Revista de Ciências da Saúde da ESSCVP, v. 3, n. 1, p. 26-32, 2011.

BRAZ, A. S. et al. Uso da terapia não farmacológica, medicina alternativa e complementar na fibromialgia. Rev. Bras. Reumatol., São Paulo, v. 51, n. 3, 2011.

BUYSSE, D. J, REYNOLDS, C. F. 3rd, MONK, T. H., BERMAN, S. R., e KUPFER, D. J. The Pittsburgh Sleep Quality Index: a new instrument for psychiatric practice and research. Psychiatry Research, 28(2), 193-213. (1989)

BUENO, R. C. et al. Exercício físico e fibromialgia. Cad. Ter. Ocup, v. 20, n. 2, p. 279-285, 2012.

FERRO, C. Correlação dos distúrbios do sono e parâmetros subjetivos em indivíduos com fibromialgia. Disponível em http://www.unioeste.br/projetos/elrf/monografias/2005/pdf/claudia.pdf 2005

HECKER, C. D. et al. Análise dos efeitos da cinesioterapia e da hidrocinesioterapia sobre a qualidade de vida de pacientes com fibromialgia: um ensaio clínico randomizado. Fisioter mov., Curitiba v. 24, n. 1, 2011. 
HIDALGO, D. C. Atualização em Fibromialgia. Medicina Legal de Costa Rica. Costa Rica, v. 30, n. 1, 2013.

SILVA, M. R. Hidroterapia no tratamento da fibromialgia. Monografia apresentada ao curso de Graduação em Fisioterapia da Faculdade de Educação e Meio Ambiente - FAEMA. Ariquemes - RO, 2014.

SILVA, K. M. O. M, et al. Efeito da hidrocinesioterapia sobre qualidadede vida, capacidade funcional e qualidade do sono em pacientes com fibromialgia. Rev Bras Reumatológica, São Paulo, v.52, n.1, p.846, 2012.

SOARES, R. L. S.; SILVA, R. O.; CARVALHO, V. C. P. Os efeitos da Fisioterapia Aquática no Tratamento da Fibromialgia. Revista Inspirar movimento e saúde, v.3, n.6, 2011.

\section{Como citar este artigo (Formato ABNT):}

SILVA, Jaqueline Santos da Silva; SPÓSITO, Auricéia Ferreira Souto; SILVA, Carla Pequeno da. A Hidroterapia no Tratamento de Indivíduos com Fibromialgia. Id on Line Rev.Mult. Psic., 2018, vol.12, n.42, Supl. 1, p. 198-210. ISSN: 1981-1179.

Recebido: 02/11/2018;

Aceito: $05 / 11 / 2018$ 